\title{
Colonic Barotrauma Causing Tension Pneumoperitoneum Following Jet Air Insufflation to the Rectum
}

\author{
Sadaf Sheikh (D), Muhammad Akbar Baig (D) \\ Department of Emergency Medicine, Aga Khan University Hospital, Karachi, Pakistan
}

Cite this article as: Sheikh S, Baig MA. Colonic Barotrauma Causing Tension Pneumoperitoneum Following Jet Air Insufflation to the Rectum. Eurasian J Emerg Med. 2018; 17 (3): 133-5.

\begin{abstract}
Rupture of colon caused by high-pressure air is a rare traumatic intra-abdominal injury. Recently, we experienced a case of colonic barotrauma followed by tension pneumoperitoneum that caused severe abdominal pain in an otherwise healthy young individual. Initially, he denied any history of trauma; later he was found to have high-pressure jet air insufflation to his rectum by the coworkers at his workplace. Radiological imaging showed high volume pneumoperitoneum. Exploratory laparotomy revealed extensive serosal lacerations. Postoperatively, the patient was discharged with uneventful recovery.
\end{abstract}

Keywords: Colon injury, barotrauma, tension pneumoperitoneum

\section{Introduction}

The use of compressed air has become increasingly common at workplaces. Its misuse is associated with injuries. Many injuries occur during pranks among the workers, and incidents have occurred in the past as a part of the practical joke. Workers do not understand that it can cause serious colonic injury, as in our case. Such injuries require adequate medical and surgical interventions to deal with their critical nature. Spraying of the perianal and anal area with excessive pneumatic force greater than the resting anal pressure and high airflow rate causes multiple site colon injury and tension pneumoperitoneum due to colon perforation. In this way, large amount of air enters through rectum in a very short time. The first case of pneumatic rupture of the colon was previously reported by Stone in 1904 (1). Here, we report an unusual and unique case of colonic barotrauma resulted in pneumatic rupture of colonic segment.

\section{Case Presentation}

A 39-year-old healthy male developed sudden onset of tense abdominal distention. He did not develop fever, melena, or vomiting. Patient was brought to our attention in the emergency room. On arrival, he was awake and complained of severe abdominal pain and respirato- ry distress. His vital signs were as follows: temperature, $37.7^{\circ} \mathrm{C}$; heart rate, 143 beats/ min; respiratory rate, 30/min; and blood pressure, $130 / 60 \mathrm{mmHg}$. Physical examination revealed a markedly distended tense abdomen with hypoactive bowel sound with generalized tenderness. Percussion produced tympanic sound. Rectal examination was unremarkable. Supine chest X-ray and upright abdomen film revealed the presence of marked pneumoperitoneum, elevation of diaphragm, and colon dilatation (Figure 1). CT abdomen showed large volume pneumoperitoneum with no contrast extravasation. Accompanying coworker initially denied any history of trauma. Perianal area did not show any sign of trauma. With the assumption of trauma-related injury, further investigation for its history was done. His coworker later confessed that the patient's rectum was deliberately sprayed using a jet-type air pipe with high pressure. The nozzle of the compressed air hose was misdirected toward his anal region and ejected high-pressure air was passed through the rectum.

He was diagnosed with colonic barotrauma secondary to insufflation of jet air through the rectum causing tension pneumoperitoneum.

His laboratory workup were as follows: hemoglobin, $14.6 \mathrm{~g} / \mathrm{dL}$; hematocrit, $42.8 \%$; white blood cell count, $10.3 \times 103 / \mu \mathrm{L}$; platelet count,

ORCID IDs of all authors: S.S. 0000-0001-7457-0012; M.A.B. 0000-0002-2830-9099. 

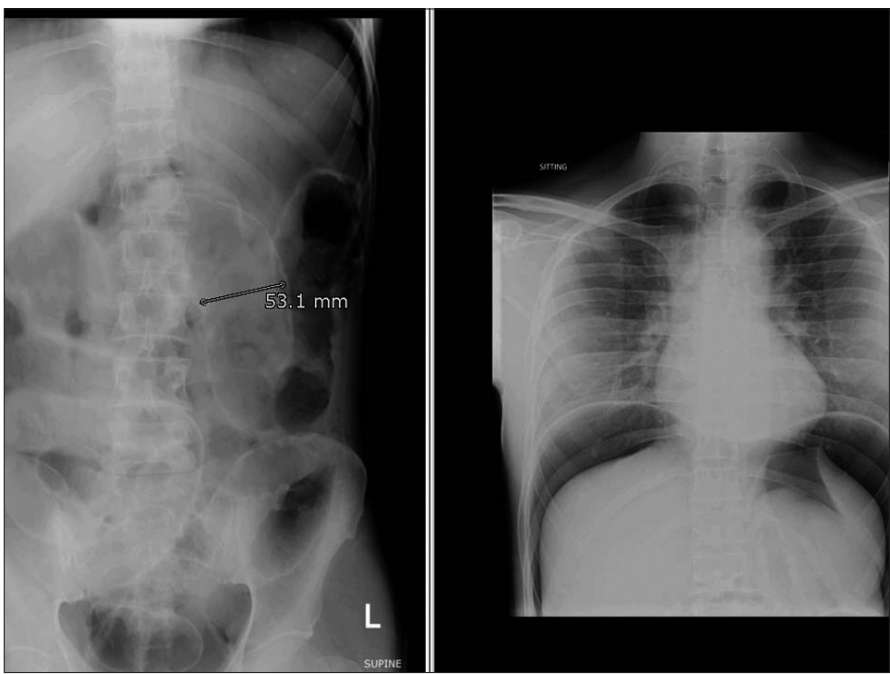

Figure 1. Supine chest X-ray and upright abdominal film showed the presence of pneumoperitoneum with distention of the colon

194×103/ $\mu \mathrm{L}$; amylase, 35 IU/L; and lipase, 28 U/L. Nasogastric tube was passed, and kept on low wall suction in the emergency room. After fluid resuscitation and analgesia, emergent exploratory laparotomy and extended right hemicolectomy with double barrel stoma was performed. Intraoperatively, there was no fecal contamination. The colon was distended with air with multiple areas of serosal lacerations and subserosal hemorrhage of the entire colon. The diaphragm, liver, spleen, and pancreas were observed to be normal. The serosal tears were repaired, and the colonic rupture was treated with ileostomy. The patient was kept under special care post-operatively and discharged few days later in stable condition with a well-functioning colostomy. Verbal consent was taken from the patient for case discussion and publication.

\section{Discussion}

External pneumatic insufflation of the colon through the anus depends on the air pressure, airflow velocity, anal resting pressure, and the distance between the source and anus (2). Under normal condition, normal resting anal pressure can prevent the insufflation of the colon from a direct external source with low air pressure $(3,4)$. Andrew has postulated that air at $3.5-8.8 \mathrm{~kg} / \mathrm{cm}^{2}$ forms a column that acts like a solid body, which forces the anal sphincter open (4). In our case, high-pressure jet insufflation can produce an air thrust of tenfold greater than the resting anal pressure. This overcomes the anal sphincter pressure, resulting in sudden inflation of the colon. The bowel wall is elastic and distensible, and it can tolerate certain amount of pressure (4). Of the four layers of the intestinal wall, mucosa is the most elastic and strongest layer and the serosa and the muscularis are the least (5). Furthermore, the funnel shape of buttocks and anus facilitates easy delivery of air. The intraluminal pressure that is required to result in colon perforation has been estimated through colonoscopic studies and has been found to be greater than $0.109 \mathrm{~kg} / \mathrm{cm}^{2}$ (5). However, previous experimental studies showed that the air pressure that leads to colon perforation was 1-4 times higher. Shiels et al. (6) found that the hydrostatic pressure to cause perforation in pig bowel is $0.16 \mathrm{~kg} / \mathrm{cm}^{2}(6-8)$.

During gradual insufflation of colon or in large bowel obstruction distal to the caecum, the caecum is the segment most prone to distention injury that is explained by the Law of Laplace (9). The caecum has the largest diameter and requires the least amount of pressure to distend (10). The anatomy of the distal colon with the firm lateral support of the rectum makes the first part of the colon to be struck by a column of pressure from external source and the bending of the sigmoid pose the recto sigmoid to rupture in pressure-related colon barotraumas (11). During rapid air distention, inability to produce a total obstruction by the bending of sigmoid and high pressure allow the flow of air proximally to the next anatomical bending such as splenic flexure and hepatic flexure and ileocaecal valve. This results in a stepwise closed loop obstruction, resulting in other site of the bowel to be injured and perforated $(2,9)$. Single site perforations are common during colonoscopic examination; however, the speed of pressure builds up following high-pressure insufflation with high flow rate resulting in multiple site colon injury $(2,9,11)$. The pathologic lesions following pneumatic insufflation depend on the resultant intraluminal pressure and include serosal hemorrhage, lacerations of the serosa and muscular coat with bulging of the mucous membrane, or complete rupture of the bowel through the serosa, muscular coat, and mucous membrane as in our case $(2,11)$.

Depending on the extent of intraluminal pressure and the presence of colonic injury, clinical manifestations vary. It can occur in single or multiple forms. Rectosigmoid region is the most common site for the perforation in particular antimesenteric wall (11). Abdominal distension and pain develops abruptly, and abdominal tenderness and rigidity are presented in case of colonic perforation. Symptoms of respiratory distress are also observed.

Tension pneumoperitoneum means that the intra-peritoneal air exerts so much pressure on the circulatory and respiratory systems that it causes respiratory distress and hypotension. Diagnosis is typical while looking at the history of exposure to compressed air and clinical manifestations. Distended colon or large amount of free air in the peritoneal cavity can be observed on imaging. Arterial blood gases showed hypoxia and respiratory alkalosis. Air pressure can compress inferior vena cava and cause hypotension and circulatory collapse.

Management of pneumatic colon injury includes two important aspects: tension pneumoperitoneum and colonic injury. After initial resuscitation, intraoperative decompression of bowel in the presence of distended bowel, resection of severely injured segment of colon, and repair of perforation with proximal diverting colostomy or enterostomy when the integrity of the bowel is in doubt should be performed $(10,11)$. Careful observation following surgery is often necessary because full-thickness perforation of the colon may have delayed presentation. Closure of stoma can be performed as early as 2-3 weeks of following creation without significant increase in complications compared with late closure, and depends on whether patient had recovered from his initial injuries, which is assessed with barium enema or sigmoidoscopic examination (11). Prognosis is favored if early surgical management is employed.

\section{Conclusion}

Patient with jet insufflation to the rectum causing tension pneumoperitoneum associated with multiple site colon injury and perforation is a unique case presentation. Laundry workers were later cautioned against playing with pneumatic tool to avoid barotrauma. Patients with jet insufflation of the rectum are at high risk of devel- 
oping bowel trauma, and as a result could develop pneumoperitoneum. Compressed air at high pressure can be hazardous and can cause significant injuries. Professionals who use them should warn their employees of the hazard and risk associated with them.

Informed Consent: Verbal informed consent was obtained from the patient who participated in this study.

Peer-review: Externally peer-reviewed.

Author Contributions: Concept - S.S., M.A.B.; Design - S.S., M.A.B.; Supervision - S.S., M.A.B.; Resources - S.S., M.A.B.; Materials - S.S., M.A.B.; Data Collection and/or Processing - S.S., M.A.B.; Analysis and/or Interpretation - S.S., M.A.B.; Literature Search - S.S., M.A.B.; Writing Manuscript - S.S., M.A.B.; Critical Review - S.S., M.A.B.

Conflict of Interest: The authors have no conflict of interest to declare.

Financial Disclosure: The authors declared that this study has received no financial support.

\section{References}

1. Stone GW. Rupture of the bowel caused by compressed air. Lancet. 1904; 2: 216. [CrossRef]
2. Sy ED, Chiu Yl, Shan YS, Ong RL. Pneumatic colon injury following high pressure blow gun dust cleaner spray to the perineum. Int J Surg Case Rep. 2015; 6: 218-21. [CrossRef]

3. Gill RS, Mangat $\mathrm{H}$, Al-Adra DP, Evans M. Hydrostatic rectosigmoid perforation: a rare personal watercraft injury. J Pediatr Surg. 2014; 46: 402-4. [CrossRef]

4. Morrison DM, Pasquale MD, Scagliotti CJ. Hydrostatic rectal injury of a jet ski passenger: case report and discussion. JTrauma. 1998; 45: 816-8. [CrossRef]

5. Woltjen JA. A retrospective analysis of cecal barotrauma caused by colonoscope air flow and pressure. Gastrointest Endosc. 2005; 61: 37-45. [CrossRef]

6. Shiels WE 2nd, Kirks DR, Keller GL, Ryckman CC, Daugherty CC, Specker $\mathrm{BL}$, et al. John Caffey award. Colonic perforation by air and liquid enemas: comparison study in young pigs. AJR Am J Roentgenol. 1993; 160 : 931-5. [CrossRef]

7. Brayko CM, Kozarek RA, Sanowski RA, Howells T. Diverticular rupture during colonoscopy. Fact or fancy? Dig Dis Sci. 1984; 29: 427-31. [CrossRef]

8. Shiels WE 2nd, Maves CK, Hedlund GL, Kirks DR. Air enema for diagnosis and reduction of intussusception: clinical experience and pressure correlates. Radiology. 1991; 181: 169-72. [CrossRef]

9. Luning TH, Keemers-Gels ME, Barendregt WB, Tan AC, Rosman C. Colonoscopic perforations: a review of 30,366 patients. Surg Endosc. 2007; 21 : 994-7. [CrossRef]

10. Loffeld RJ, Engel A, Dekkers PE. Incidence and causes of colonoscopic perforations: a single-center case series. Endoscopy. 2014; 43: 240-2. [CrossRef]

11. Brown RK, Dwinelle JH. Rupture of the colon by compressed air: report of three cases. Ann Surg. 1942; 115: 13-20.[CrossRef] 